\title{
Comprehensive Beam Jitter Study for the Commissioning of the Intermediate Matching Section and Drift Tube Linac at Ground Test Accelerator*
}

\author{
D. S. Barr, J. D. Gilpatrick, \\ Los Alamos National Laboratory \\ MS H808, Los Alamos, NM 87545
}

\section{Abstract}

An experiment on the Ground Test Accelerator (GTA) for the Neutral Particle Beam (NPB) at Los Alamos commissioned the intermediate matching section (IMS) and a single $3.2-\mathrm{MeV}$ drift tube linac (DTL). A diagnostic platform or D-plate was used at the output of the DTL in order to measure various beam parameters. The D-plate and other diagnostic devices located in the IMS, provided measurement of the horizontal and vertical beam position, current, energy, and output phase. These instruments were installed to perform a complete beam jitter analysis based on the current beamline configuration to better understand the causes of any jitter sources as well as to prepare for the initial design of future feedback control systems. The study explored all types of jitter for various beamline configurations. Both interpulse jitter (jitter from pulse to pulse) and intrapulse jitter (jitter within each macropulse) were investigated. Spectral and statistical time analyses were used. Spectral analysis was employed to gain an understanding of the spectral contributions of various jitter sources to determine the degree of correction possible. Statistical time analysis gave a good overall representation of the jitter magnitude and allowed easy comparison of jitter for different beamline configurations, as well as an easy method for determining consistent problems.

\section{INTRODUCTION}

Jitter data were collected during the GTA Experiment 2A run in October and November of 1992. The beamline consisted of an $\mathrm{H}^{-}$ion source and injector, followed by an RFQ (Radio Frequency Quadrupole), an IMS used to match the RFQ output to the DTL input in the transverse and longitudinal phase space), a DTL, and a D-plate. The D-plate contained various diagnostic devices, including three microstrip beam probes (MBP), a toroid, three Faraday cups, and a slit and collector (for transverse emittance measurements). The measurements utilized six MBPs, four toroids, and a Faraday cup [1]. MBP \#1, \#2, and \#3 were located in the IMS, while MBP \#4, \#5, and \#6 were located on the D-plate. The MBPs on the D-plate were separated by drifts, thus allowing beam trajectory angle and time-of-flight energy measurements. Toroids \#1 and \#2 were located just before and after the RFQ, toroid \#3 was located in the IMS, and toroid \#4 was located on the D-plate. The DTL output phase measurements were

\footnotetext{
*Work supported and funded by the US Department of Defense, Army Space and Strategic Defense Command, under the auspices of the US Department of Energy.
}

obtained by comparing the phase of the DTL electric fields to the phase of the MBP \#4 signal. Both the MBPs and the toroids produce measurements of the beam current. The MBP current measurements (referred to as intensity) produces an output proportional to the peak bunch current at any given point in time. The toroid measures the average beam current integrated over time. Note that the data presented here are just a subset of the total data taken.

\section{II . MEASUREMENT DESCRIPTION}

Intrapulse data, which are composed of multiple data points taken within a single macropulse were taken. Using a 4channel, 12-bit digitizer, we were able to take data at 5 megasamples per second. A $2.0-\mathrm{MHz}$ analog filter was used on the input of the digitizer to remove any aliased frequency spectra. Each macropulse was roughly $300 \mu \mathrm{sec}$ long, and therefore 1500 points per macropulse were taken. We collected 25 consecutive macropulses on each of the four digitizer channels for many different accelerator configurations. Various combinations of beam position, current, energy, and DTL output phase were taken synchronously. Data presented were taken with all cavities and devices operating in-time (the RFQ, both IMS cavities, and the DTL), and with the steering quads set to their nominal design values.

\section{PROCESSING OF DATA}

A brief description of the algorithm used to produce the power spectrum plots is given below:

1) Conversion from raw digitizer counts to appropriate units (ma, mm, MeV, degrees)

2) Removal of data points outside the macropulse

3) Removal of dc component

4) Data was windowed using Von Hann window

5) The FFT of the data was taken

6) The magnitude of the data was taken

7) Each value was divided by the number of data points

8) The magnitude of each value was squared

9) Each value of the spectrum was scaled for energy lost in windowing and display of one-sided spectrum

10) The deterministic noise components created by the digitizer were removed

11) The ensemble average of the various spectra was taken

12) The square root was taken to get the root-mean square (rms). 


\section{DATA ANALYSIS}

Figures 1-6 show the rms ensemble averages of the power spectrum of 25 consecutive macropulses for the intrapulse data. Table 1 lists each of the measurements along with the average mean and standard deviation for the 25 consecutive macropulses. The mean is the ensemble average of time averages for each macropulse. The standard deviation is the square root of the ensemble average of the time-variance of each macropulse [2], [3]. The mean and standard deviation of each interpulse data set is also given in Table 1.

\section{RESULTS AND CONCLUSIONS}

In most of the position measurements there is an interesting clump of frequency spectra located around 1.95 $\mathrm{MHz}$. These spectra show up in the microstrip intensity measurement, but not in any of the others. Because the microstrip intensity measurements are dependent on beam position and the toroid measurements are not, it can be inferred that this is a beam position phenomenon.

There is a fairly clear peak at about $0.95 \mathrm{MHz}$ in all of the position and current measurements except the first toroid (not shown). Because this is the only device preceding the RFQ, it can be inferred that this peak is caused by the RFQ. There is an interesting peak at $0.55 \mathrm{MHz}$, which is seen in plots of the toroid, the energy, and the phase.

Note the large degree of low-frequency movement in most of the plots. Doubtless some of this is leakage from the $\mathrm{dc}$ component, but most of it is real beam jitter. It can be seen that the intrapulse energy and phase jitter are small, typically $0.048 \% \Delta \mathrm{W} / \mathrm{W}$ and 0.23 degrees in phase. The intrapulse current jitter was measured typically at less than $1 \%$.

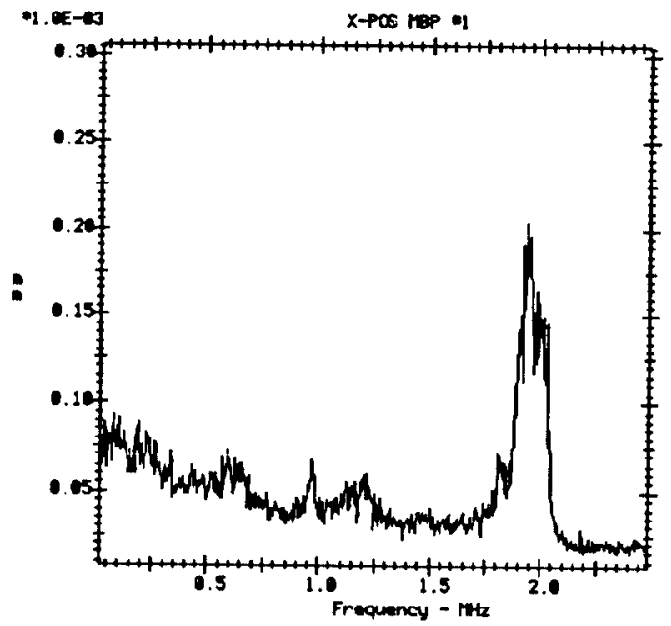

Figure 1 - Averaged Power Spectrum

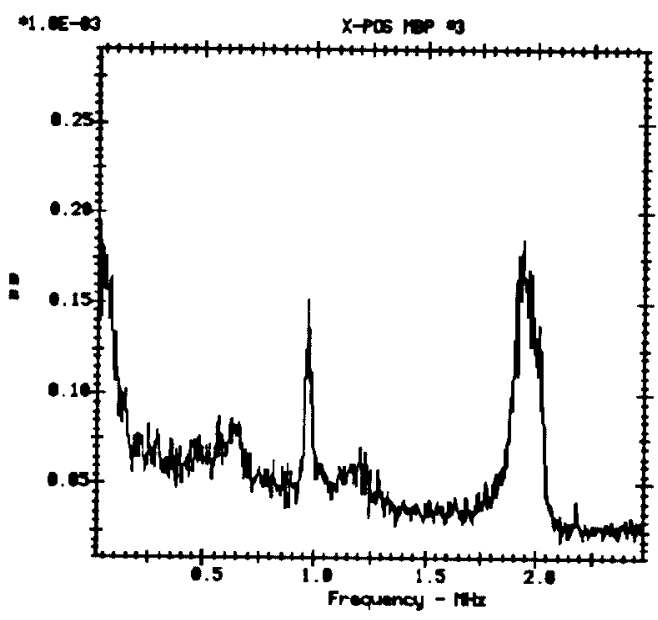

Figure 2 - Averaged Power Spectrum

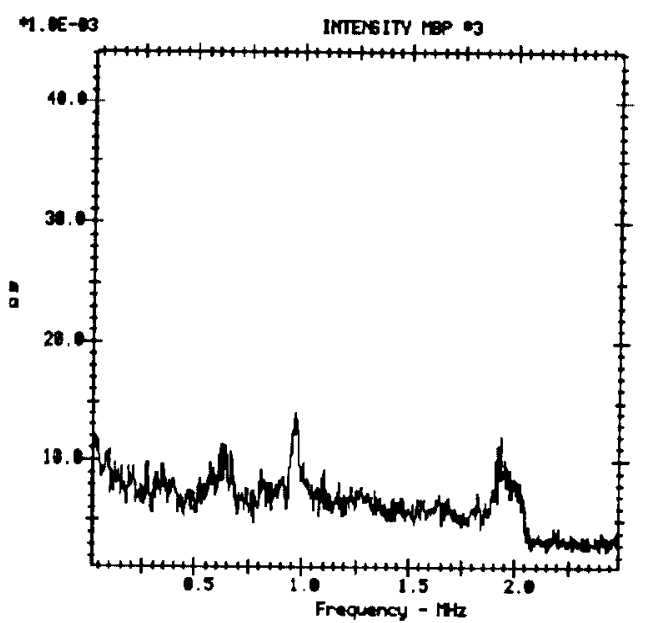

Figure 3 - Averaged Power Spectrum

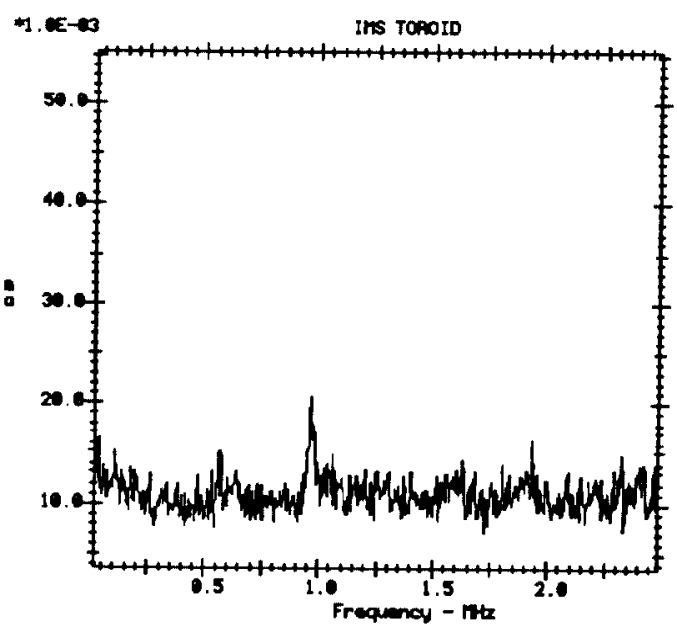

Figure 4 - Averaged Power Spectrum 


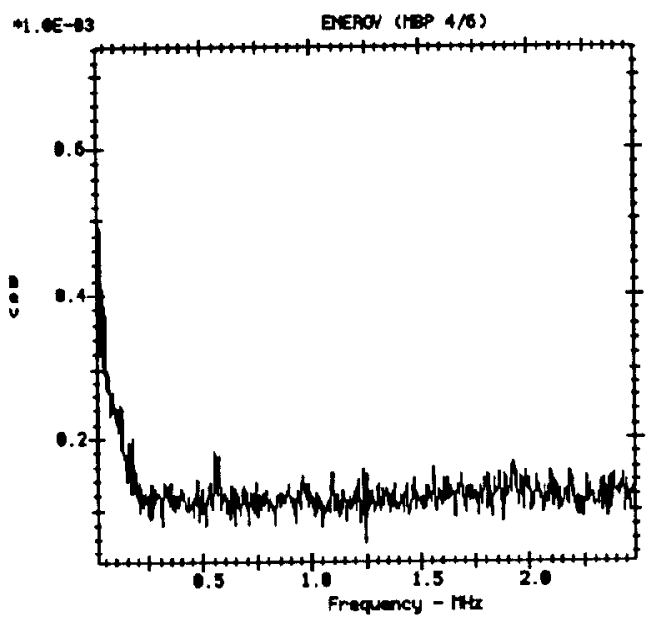

Figure 5 - Averaged Power Spectrum

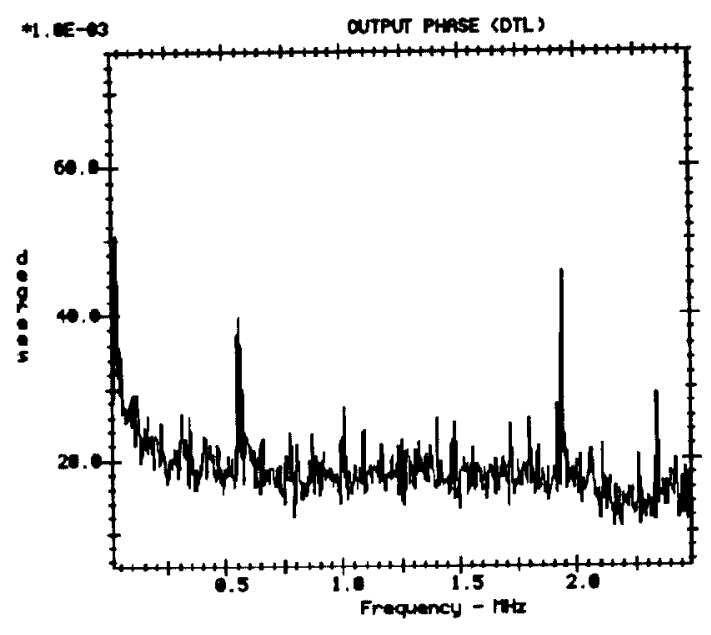

Figure 6 - Averaged Power Spectrum

Table 1

Time-Data Statistics for Intrapulse Data

\author{
Measurement Device \\ Horizontal position at MBP \#1 \\ Horizontal position at MBP \#3 \\ Intensity at MBP \#3 \\ Current at toroid \#3 (IMS) \\ Energy from MBP \#4 to \#6 \\ DTL output phase
}

\begin{tabular}{l} 
Mean \\
\hdashline $341.427 \mu \mathrm{m}$ \\
$61.377 \mu \mathrm{m}$ \\
$33.641 \mathrm{ma}$ \\
$32.544 \mathrm{ma}$ \\
$3.173 \mathrm{MeV}$ \\
$60.826 \mathrm{dcgrees}$
\end{tabular}

St. Dev.

$0.564 \mu \mathrm{m}$

$0.611 \mu \mathrm{m}$

$0.190 \mathrm{ma}$

$0.326 \mathrm{ma}$

$1.525 \mathrm{Kev}$

0.232 degrees

\section{REFERENCES}

[1] J.D. Gilpatrick, F.D. Wells, R. Martinez, K.F. Johnson, R.E. Meyer, S. Lloyd, J.F. Power, G.H. Neuschaefer, R.B. Shurter, "Measurements and Performance of a Microstrip Beam Probe System," 1991 Particle Accelerator Conference Proceedings, San Francisco, CA, May 6-9, 1991, Los Alamos National Laboratory LAUR-1428.

[2] F.G. Stremler, Introduction to Communication Systems, 2nd edition, Addison-Wesley Publishing Company, Rcading, Mass., 1982, p. 170.

[3] W.H. Press, B.P. Flannery, S.A. Teukolsky, W.T. Vetterling, Numerical Recipes: The Art of Scientific Computing, Cambridge University Press, Cambridge, England, 1986, p. 484. 